\title{
Surgical criteria for femoroacetabular impingement syndrome: a scoping review
}

\author{
Scott Peters, ${ }^{1}$ Alisha Laing, ${ }_{1}^{1}$ Courtney Emerson, ${ }^{1}$ Kelsey Mutchler, ${ }_{1}^{1}$ Thomas Joyce, ${ }^{1}$ \\ Kristian Thorborg, ${ }^{2}$ Per Hölmich, ${ }^{2}$ Michael Reiman ${ }^{1}$
}

- Additional material is published online only. To view please visit the journal online (http://dx.doi.org/10.1136/ bjsports-2016-096936).

${ }^{1}$ Duke University, Durham, North Carolina, USA

${ }^{2}$ Sports Orthopedic Research Center-Copenhagen (SORC-C), Copenhagen University Hospital, Amager-Hvidovre, Hvidovre, Denmark

\section{Correspondence to}

Dr Michael Reiman, Duke University, 2200 W Main St, Durham, NC 27708, USA: reiman.michael@gmail.com

Accepted 13 January 2017 Published Online First 20 February 2017

\section{ABSTRACT}

Background The purpose of this review was to analyse and report criteria used for open and arthroscopic surgical treatment of femoroacetabular impingement syndrome (FAIS).

Methods A librarian-assisted computer search of Medline, CINAHL and Embase for studies related to criterion for FAIS surgery was used in this study. Inclusion criteria included studies with the primary purpose of surgery or surgical outcomes for treatment of FAIS with and without labral tear, and reporting criteria for FAIS surgery.

Results Diagnostic imaging was a criterion for surgery in $92 \%$ of the included studies, with alpha angle the most frequently reported ( $68 \%$ of studies) criterion. Reporting of symptoms was a criterion for surgery in 75\%, and special tests a criterion in 70\% of studies. Range-of-motion limitations were only a required criterion in $30 \%$, only $12 \%$ of studies required intra-articular injection and $44 \%$ of studies described previously failed treatment (non-surgical or physiotherapist-led rehabilitation) as a criterion for surgery. Only $56 \%$ of included studies utilised the combination of symptoms, clinical signs and diagnostic imaging combined for diagnosis of FAIS as suggested by the Warwick Agreement on FAIS meeting.

Conclusion Diagnostic imaging evidence of FAIS was the most commonly reported criterion for surgery. Only $56 \%$ of included studies utilised the combination of symptoms, clinical signs and diagnostic imaging for diagnosis of FAIS as suggested by the Warwick Agreement on FAIS meeting, and only $44 \%$ of studies had failed non-surgical treatment (and 18\% a failed trial of physiotherapy) as a criterion for surgery.

\section{INTRODUCTION}

Femoroacetabular impingement (FAI) has been proposed as a common cause of hip pain and potential future osteoarthritis. ${ }^{1}$ Recently, the term 'FAI syndrome' (FAIS) has been suggested to more accurately encompass the heterogeneous nature of this condition. $^{2}$ A recent consensus statement ${ }^{3}$ recommended the definition of FAIS as 'a motion-related clinical disorder of the hip with a triad of symptoms, clinical signs, and imaging findings. It represents a premature contact between the proximal femur and the acetabulum'. Cam morphology (aspherical femoral head) and pincer morphology (excessive acetabular coverage of femoral head) are the two most common morphological variations of FAIS. ${ }^{14}$ Mix morphology (cam and pincer) has also been described. ${ }^{5}$ Reported prevalence of cam morphology in the general population or selected subgroups ranges from 5\% to $75 \%$, although the actual prevalence estimates cannot be determined due to insufficient high quality data and heterogeneity in morphology definition. ${ }^{6}$

Surgical intervention for FAIS continues to escalate, with a 25-fold increase from 2006 to 2013, ${ }^{7}$ and further rises are anticipated. ${ }^{8}$ Additionally, research that includes surgical intervention for FAIS has drastically increased over the past several years; analysing the variability of data within the literature can help determine appropriate surgical intervention criteria for future research. Despite this increasing prevalence, the criteria for diagnosis and surgical treatment of FAIS appear to be highly variable. ${ }^{89}$ Inconsistencies in FAIS surgical outcome reporting have been suggested ${ }^{9}$ and, combined with variable criteria for surgery, are a major concern in the management of hip dysfunction. ${ }^{210}$

The relevant literature regarding FAIS diagnosis and surgery is both vast and diverse. In such cases, scoping reviews have been recommended. ${ }^{11}$ Scoping reviews have been described as a process to map the existing evidence or literature, ${ }^{11}$ and useful frameworks to collate and summarise information on a broad topic. ${ }^{12}$ Several key differences exist between scoping and systematic reviews. Systematic reviews typically have a focused research question with narrow parameters, whereas scoping reviews tend to be broader. Furthermore, while systematic reviews formally assess the quality of studies and generate a conclusion related to the focused question, scoping reviews are used to identify parameters and gaps in a body of literature. Because this review incorporated many different types of studies to identify appropriate parameters regarding surgical treatment for FAIS, a scoping review was chosen.

The purpose of this review was to report the explicit criteria used in research studies on both open and arthroscopic surgical treatment of FAIS. We hypothesised that diagnostic imaging evidence of FAIS would be the primarily reported criterion for surgery. However, we expected to find vast differences among studies for the other criteria used to identify appropriate candidates for surgical intervention.

\section{METHODS \\ Identification of studies}

This review was originally registered as a systematic review with Prospero (CRD42016033373) after completion of preliminary search and initiation of data extraction. The heterogeneity and breadth of study types in inclusion criteria required changing 
the review to a scoping review to more accurately reflect the current status of surgical criteria for FAIS surgery.

We conducted a librarian-assisted computer search of Medline, CINAHL and Embase for studies on the criterion for FAI surgery. The search was performed on 3 November 2015 from the inception time of each database. Our search strategy was as follows: electronic databases (Medline, Embase and CINAHL) were searched for surgical FAIS studies. The search strategy used the following terms: (Hip[Mesh] OR Hip[tiab] OR hips [tiab] OR 'Hip Joint' “ [Mesh] OR 'coxofemoral joint' [tiab]) AND ('Femoroacetabular Impingement'[Mesh] OR 'femoroacetabular impingement'[tiab] OR 'Femoral acetabular impingement'[tiab] OR FAI[tiab] OR femoral impingement OR impingement [tiab] OR 'labral tear'” [tiab] OR 'labral tears'[tiab] OR acetabular tear OR acetabular tears) AND ('Orthopedics'[Mesh] OR Surgery[tiab] OR 'surgery' [Subheading]) AND (diagnosis[tiab] OR diagnostic[tiab] OR diagnosed[tiab] OR diagnosis[MeSH] OR diagnosis[sh] OR indication[tiab] OR indications[tiab] OR cross-sectional studies[Mesh] OR cross-sectional[tiab]) NOT (Editorial[ptyp] OR Letter[ptyp] OR Case Reports[ptyp] OR Comment[ptyp]) NOT (animals[mh] NOT humans[mh]).

\section{Eligibility criteria}

The inclusion criteria for this scoping review included the following: (1) studies of subjects treated with arthroscopic or open hip surgery for the treatment of FAIS; (2) studies completed with the primary purpose of surgery or surgical outcomes for the treatment of FAIS; and (3) studies reporting criterion for
FAIS surgery. Exclusion criteria were as follows: (1) studies of subjects with hip dysplasia, slipped capital femoral epiphysis or Legg-Calve-Perthes disease; (2) studies of subjects receiving revision hip arthroscopy or revision open surgery; (3) studies with subjects undergoing periacetabular osteotomy interventions; (4) studies with subjects undergoing surgery for procedure other than FAIS (eg, ligamentum teres tear, osteoarthritis) with or without labral repair; (5) case studies and case series $n<10$; and (6) studies not reported in English.

\section{Screening, eligibility and inclusion}

Two independent reviewers completed the title and abstract screen, followed by reviewers independently completed title, abstract and full text screen. Discrepancies between the two independent reviewers were resolved by a third reviewer. The review process is described below in figure 1 , including reasons for exclusion.

\section{Data extraction}

Data were collected independently from the included studies by two reviewers. The data elements collected consisted of publication year, study size, percent of cam and pincer reported FAIS, and surgical criteria for the treatment of FAIS. The surgical criteria collected included subjective history of patient symptoms, clinical signs (eg, range of motion measurements, special tests and previous non-surgical treatments) and diagnostic imaging. ${ }^{3}$ The data elements were verified by another reviewer,

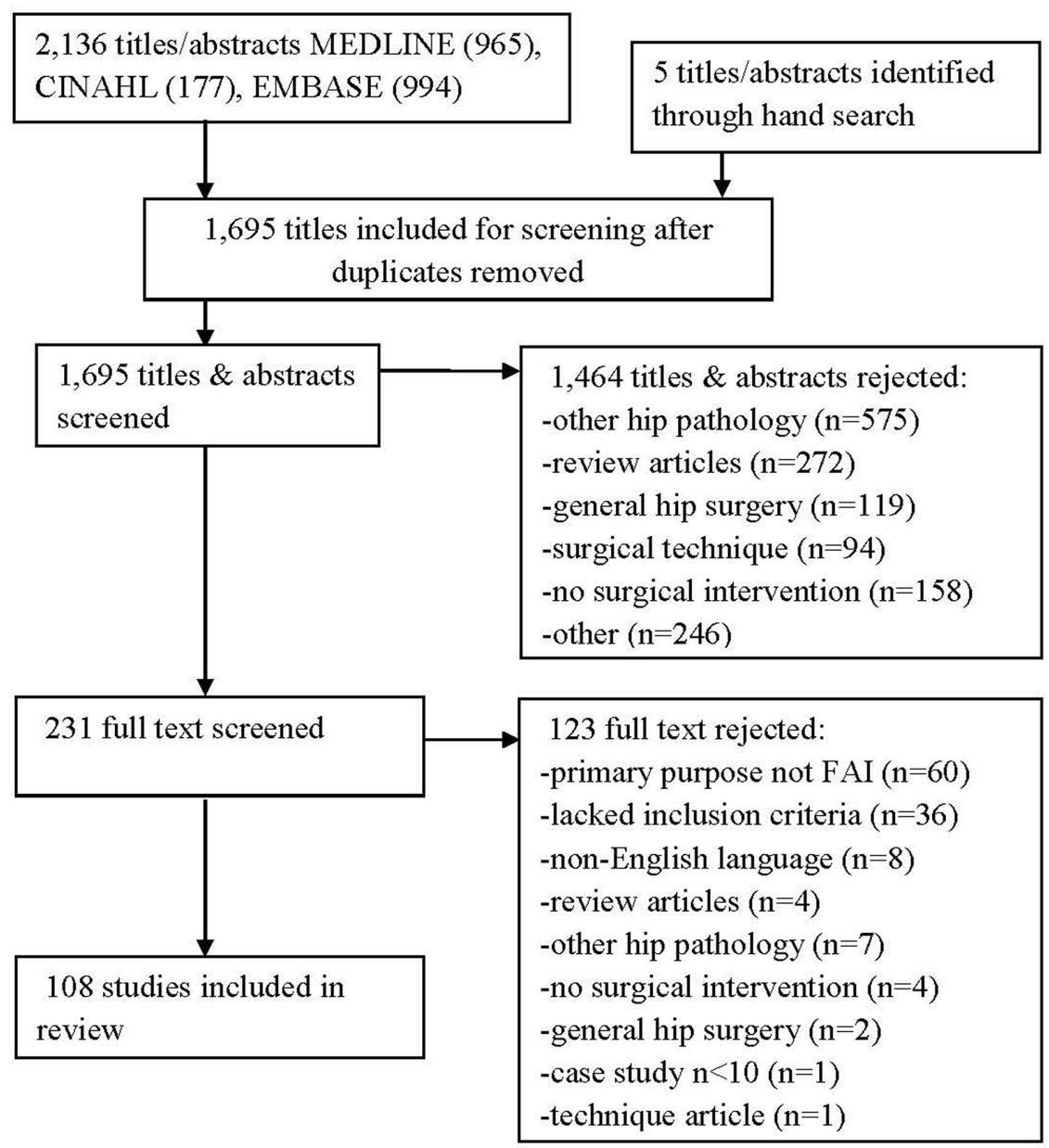

Figure 1 Flow diagram for study inclusion. FAl, femoroacetabular impingement. 


\begin{tabular}{ll}
\hline Table 1 Patient demographics & \\
\hline Patient demographics & \\
\hline Patients, $\mathrm{n}$ & 10001 \\
\hline Hips, $\mathrm{n}$ & 10618 \\
\hline Average \% male & 56.5 \\
\hline Average mean age & 33.0 years \\
\hline Total reporting type of lesion & 70 studies \\
\hline Total hips & 6019 \\
\hline$\%$ Cam & 40 \\
\hline$\%$ Pincer & 8.4 \\
\hline$\%$ Mixed & 51.7 \\
\hline
\end{tabular}

and disagreements were resolved through discussion. Data elements for patient demographics, reported surgical criteria (eg, symptoms, clinical signs and diagnostic imaging), type of FAIS and diagnostic imaging criteria utilised in the various studies were analysed and grouped accordingly. We also determined the prevalence of reporting criteria according to published criteria for the diagnosis of FAIS suggested by Nepple et $a l^{13}$ and the recent FAIS consensus statement ${ }^{3}$ to determine reporting consistency. Prevalence of reported detailed diagnostic imaging criteria detail as suggested by Nepple et $a l^{13}$ is also provided.

\section{RESULTS}

\section{Study identification}

Our initial literature search yielded 2136 studies, of which 108 met the inclusion criteria for this review. There was excellent inter-rater agreement among reviewers in title and abstract review $(\mathrm{k}=0.79)$ as well as excellent agreement in full-text screening $(\mathrm{k}=0.80)$.

\section{Study demographics}

All included studies were conducted since 2004. Expanded characteristics and demographics of each study included can be seen in table 1.

\begin{tabular}{lc}
\hline $\begin{array}{l}\text { Table } 2 \\
\text { criteria }\end{array}$ & Criteria of all studies compared against Nepple et al $^{13}$ \\
\hline Frequency of diagnostic criteria from Nepple et ${ }^{13}$ & \\
\hline Criteria & Studies reporting criteria \\
\hline Hip pain $>3$ months & $26(24 \%)$ \\
\hline $\begin{array}{l}\text { No clinical evidence of inflammatory arthritis } \\
\text { changes }\end{array}$ & $69(64 \%)$ \\
\hline Hip internal rotation $<20$ in $90^{\circ}$ hip flexion & $6(6 \%)$ \\
\hline $\begin{array}{l}\text { Lateral centre edge (CE) angle }>20^{\circ} \\
\text { Alpha angle }>60^{\circ}, \mathrm{CE} \text { angle }>40^{\circ} \text { or presence of }\end{array}$ & $72(67 \%)$ \\
acetabular retroversion & $2(2 \%)$ \\
\hline $\begin{array}{l}\text { Alpha }>60^{\circ} \\
\text { CE angle }>40^{\circ}\end{array}$ & $8(7 \%)$ \\
\hline $\begin{array}{l}\text { Acetabular retroversion (stating crossover sign) } \\
\text { Diagnostic injection or MRI indicating presence of } \\
\text { intra-articular pathology or labral damage }\end{array}$ & $62(57 \%)$ \\
\hline
\end{tabular}

\section{Reported outcomes}

The total percentage of studies utilising the primary criterion for surgery investigated is shown in figure 2 with clinical signs being represented by range of motion and special tests. As shown in this figure, diagnostic imaging was the most prevalently reported criterion for surgery (92\% of studies).

As previously mentioned, two standards for the diagnosis of FAIS have been reported. ${ }^{3} 13$ The initial proposal by Nepple et $a l^{13}$ proposed specific criteria for diagnosis of FAIS. The number and frequency of studies reporting each of these proposed criterion are provided in table 2.

More recently, the 2016 Warwick Agreement on FAIS ${ }^{3}$ has been proposed, resulting from a 1-day consensus meeting with the participation of 22 panel members and one patient from nine countries and five different specialties. In this consensus paper, three distinct criteria were recommended for FAIS diagnosis: symptoms (eg, groin pain and mechanical symptoms), clinical signs (eg, positive special tests and limited motion) and diagnostic imaging. The number of studies reporting all three of these criteria for FAIS diagnosis for surgery was 60

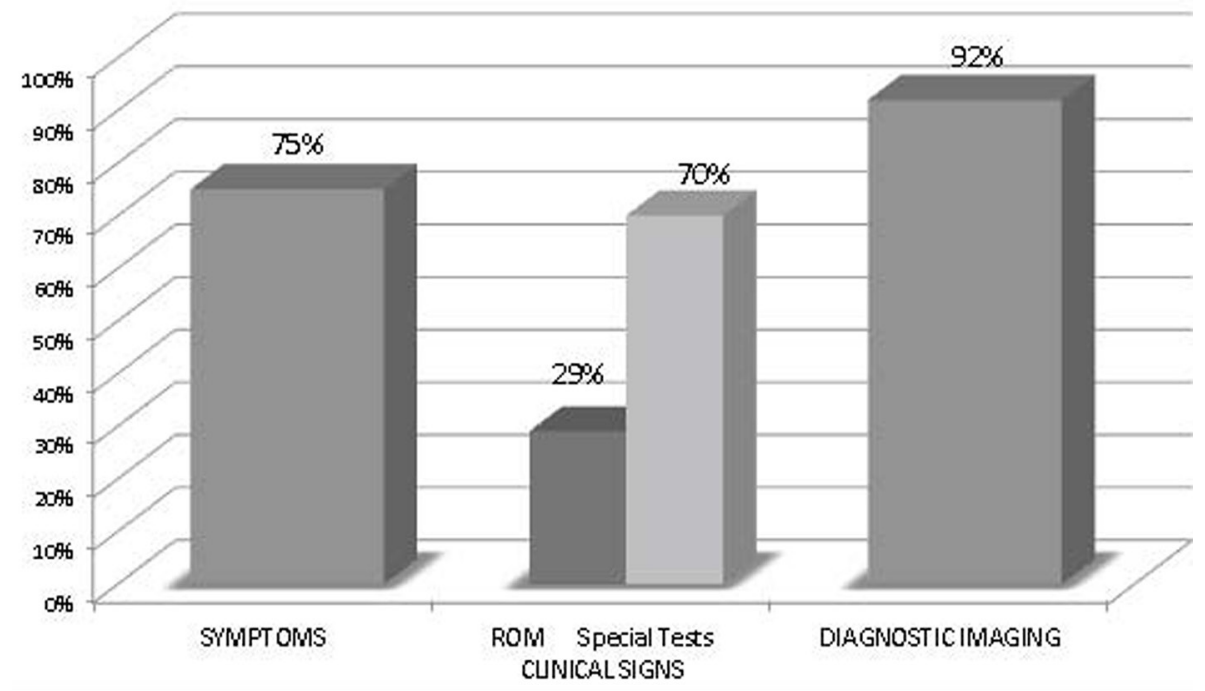

Figure 2 Summary of symptoms, clinical signs and diagnostic imaging criteria prevalence for surgical criteria in included studies. ROM, range of motion. 


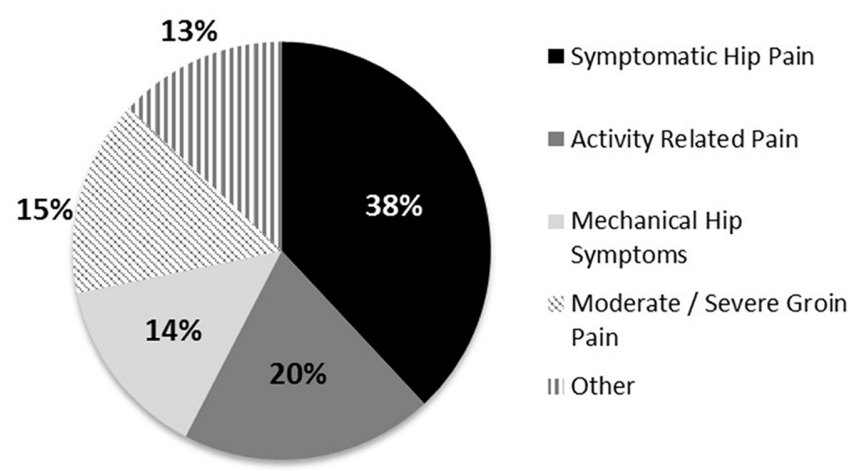

Figure 3 Summary of reported patient symptom criteria prevalence for surgical criteria in included studies.

(56\%), the number utilising two of three of these criteria was $37(35 \%)$ and the number utilising only one of three of these criteria was 10 (9\%) (see online supplementary appendix 1).

\section{Symptoms}

Eighty-one (75\%) studies reported using a defined symptom as a surgical criterion, with 35 (32\%) of the studies reporting criteria grouped as 'symptomatic hip pain', 18 (17\%) were grouped as 'activity-related pain' and 13 (12\%) included symptoms consistent with 'mechanical hip pain' (figure 3 ). Due to a wide variety of reported criteria such as 'clinical symptoms' and 'disabling pain', grouping of symptoms and criteria was required for analysis. Duration of symptoms was also reported as a surgical criterion in $30(28 \%)$ studies with two studies reporting greater than 1 year, 13 reporting 6 months symptomatic, 11 reporting 3 months symptomatic and four reporting less than 3 months symptomatic.

\section{Intra-articular injection}

Relief of symptoms after intra-articular injection is suggested as a 'symptom' category for FAIS diagnosis per recent 2016 Warwick Agreement. Only 13 studies (12\%) reported on intra-articular injection as a surgical criterion. Most of these 13 studies utilised a combination of MRI and/or MR arthrography (MRA) with intra-articular injection, while only three $(3 \%)$ required an intra-articular hip injection that relieved pain and symptoms without use of either MRI or MRA.

\section{Clinical signs}

Clinical signs were reported across 80 (74\%) studies in this review (online supplementary appendices 1 and 2 ).

The clinical signs reported across these studies were grouped accordingly (range of motion, special tests).

\section{Range of motion}

Range of motion measurements were utilised as surgical criteria in $32(30 \%)$ studies with a wide variety of measurement reporting. As shown in figure 4, $12(11 \%)$ studies reported a vaguely defined method of 'measured', whereas 12 (11\%) reported 'flexion and internal rotation were decreased'. Six (6\%) studies reported 'internal rotation less than $20^{\circ}$ ' and seven (6\%) reported 'internal rotation decrease' only.

\section{Special tests}

Special tests were reported as surgical inclusion criteria in 76 $(70 \%)$ studies with a primary focus on the re-creation of pain in the symptomatic position (figure 5). Many special tests have

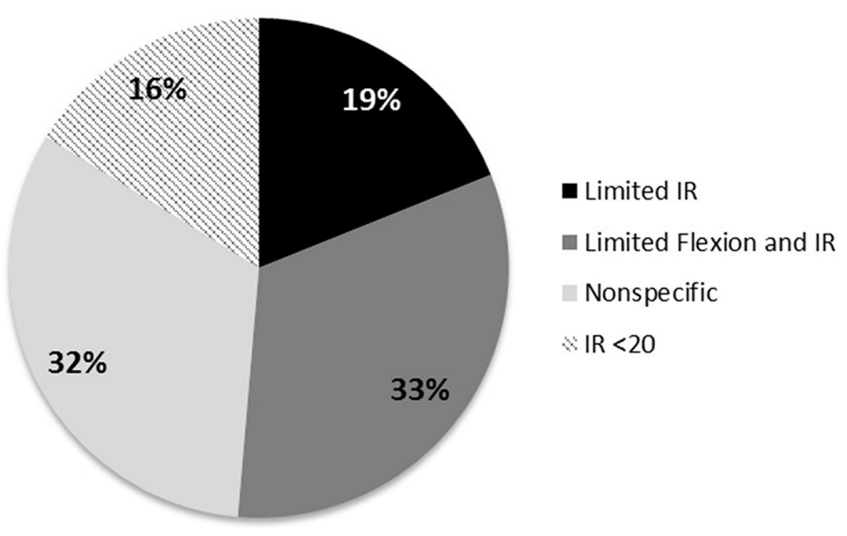

Figure 4 Summary of reported clinical signs criteria prevalence for surgical criteria in included studies. IR, internal rotation.

multiple terminologies and are used interchangeably in both the literature and the clinical setting. Because of these discrepancies, impingement tests (specifying anterior impingement tests) were grouped together with the flexion, adduction, internal rotation (FADIR) test, while unspecified impingement tests were categorised separately. By utilising this categorisation, 38 (35\%) studies described a 'positive anterior impingement test' or 'FADIR', while 39 (36\%) studies described a 'positive impingement test'. Lesser-utilised tests included the FABER, ${ }^{14} \log$ roll, ${ }^{2}$ labral stress test ${ }^{2}$ and the McCarthy test. ${ }^{1}$

\section{Diagnostic imaging}

Diagnostic imaging was widely reported throughout the studies with 99 (92\%) studies reporting it as surgical inclusion criteria; alpha angle measurement was the most commonly reported criterion (68\% of all studies) (tables 2 and 3, figure 6; see online supplementary appendix 3). Of the 73 studies utilising alpha angle as a criterion, two studies (3\% of studies utilising alpha angle) included a cut-off of $>60^{\circ}$ as recommended by Nepple et $a l .{ }^{13}$ Additionally, of the 55 studies where centre edge angle was utilised as surgical criteria, eight (15\% of studies utilising centre edge angle) reported an objective value of $>39^{\circ}$. Of the 63 studies utilising either MRI or MRA, 42 (64\%) required an MRI and $21(32 \%)$ required an MRA.

Interestingly, $10(15 \%)$ of these studies required both MRI/ MRA and a pain relieving hip injection. In fact, multiple imaging methods were utilised in $86(80 \%)$ of all studies in this review, with $24 \%$ of studies utilising all four imaging criteria reported in

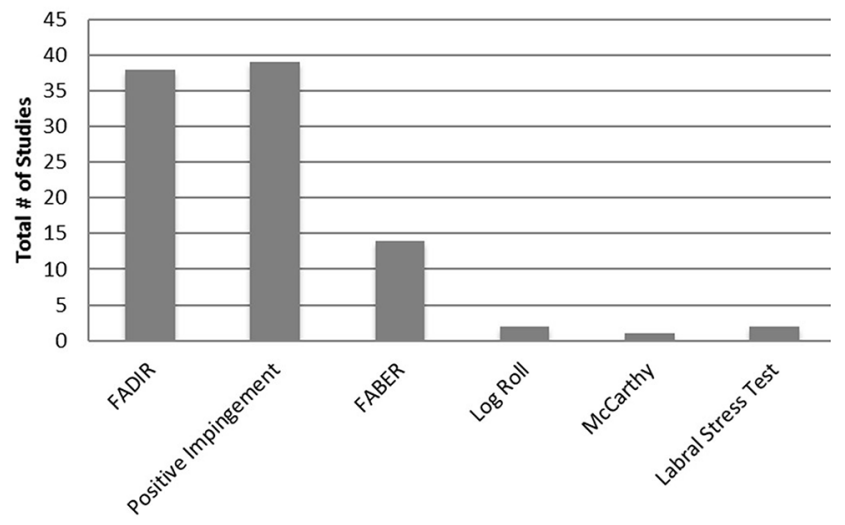

Figure 5 Summary of reported special tests prevalence for surgical criteria in included studies. 
Table 3 Summary of diagnostic imaging findings for pincer impingement values for surgical criteria in included studies

\begin{tabular}{lcc}
\hline \multicolumn{2}{l}{ Diagnostic findings for pincer impingement } \\
\hline Centre edge angle & \\
Reported & 55 & $51 \%$ \\
$\quad$ Non-specific & 47 & $44 \%$ \\
$\quad$ Specific $\left(>39^{\circ}\right)$ & 8 & $7 \%$ \\
Crossover sign & & \\
Reported & 62 & $57 \%$ \\
\hline
\end{tabular}

this review (MRI/MRA, alpha angle, centre edge angle and crossover sign), 28\% utilising three of these imaging criteria, 19\% of studies utilising two of these criteria, 21\% utilising a single imaging criterion and $8 \%$ not using an imaging criterion for the diagnosis of FAIS (see online supplementary appendix 3 ). In the studies utilising a single criterion, the primary criterion was MRI or MRA in $15(65 \%)$ of the studies.

\section{Failed prior non-surgical treatment for FAIS}

Failed prior non-surgical treatment was reported in 47 (44\%) of all studies (see online supplementary appendix 3). The definition of failed prior treatment was poorly described, most notably listing a general statement of 'non-surgical' or 'conservative' treatment. Ten (9\%) of all 108 studies reported having treatment for 6 months, 10 (9\%) for 3 months, five (5\%) for less than 3 months and $22(20 \%)$ for an unspecified amount of time. Sixteen (15\%) of all studies reported receiving non-steroidal anti-inflammatory drugs (NSAIDs), 21 (19\%) activity modification and 19 (18\%) formal physiotherapy-led rehabilitation, and $18(17 \%)$ studies reported utilising a general non-descript prior treatment that was not successful. Nine studies (8\%) received a combination of NSAIDs, activity modification and physiotherapy-led rehabilitation.

In summary, criteria for surgical intervention regarding FAIS are diverse and inconsistently reported. The most commonly reported surgical criteria were symptomatic hip pain of greater than 6 months' duration (symptom), decreased hip flexion and internal rotation range of motion (clinical sign), a positive impingement sign (clinical sign), alpha angle greater than $50^{\circ}$ (diagnostic imaging) and a positive crossover sign (diagnostic imaging). While these present as the most commonly utilised surgical criteria, it is important to realise that these were not

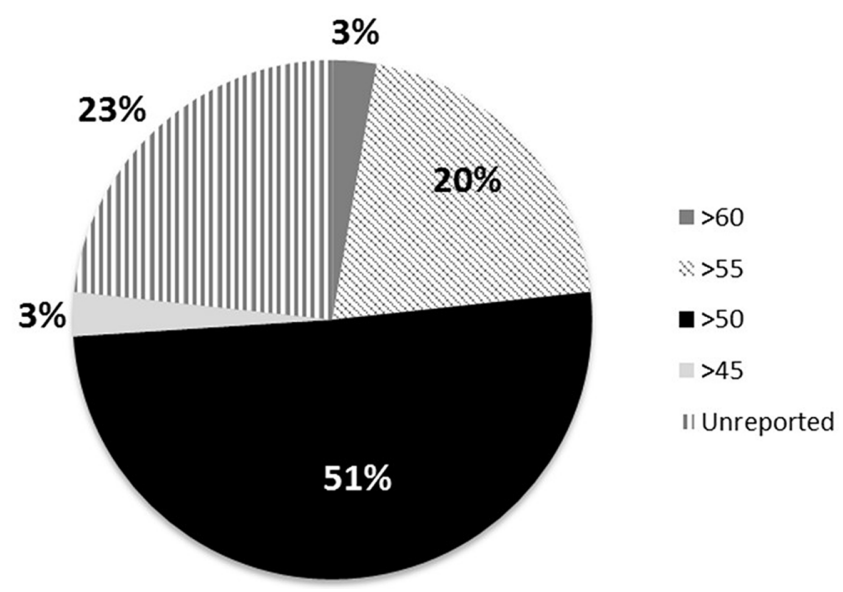

Figure 6 Summary of alpha angle diagnostic imaging values for surgical criteria in included studies. consistently reported group criteria in the same studies but rather the most commonly reported across all studies.

\section{DISCUSSION}

We sought to analyse and report the inclusion criteria used for open and arthroscopic surgical treatment of FAIS. Similar to previous findings in a smaller sample size of surgical outcome studies only, ${ }^{9}$ this review found significant inconsistency among the reporting and specific criteria required for surgical treatment of FAIS. As reported previously, such heterogeneity in reported surgical criteria questions whether the same condition is being treated across all studies. ${ }^{10}$

Two recent guidelines ${ }^{313}$ have been proposed to assist with the comprehensive clinical presentation of FAIS. ${ }^{2}$ In 2012, Nepple et $a l^{13}$ proposed a comprehensive list of inclusion and exclusion criteria for the diagnosis of FAIS utilising a multidimensional approach as outlined in table 2. Consistent with the results of the current review, the Nepple et al criteria ${ }^{13}$ are predominantly diagnostic imaging criteria. The Nepple $e t a l^{13}$ criteria also lack clarity in reporting of non-surgical treatment details and specific range of motion limitations. Utilising the three criteria for diagnosis of FAIS (symptoms, clinical signs and diagnostic imaging) by the 2016 Warwick Agreement on FAIS, ${ }^{3}$ we found that only $56 \%$ of the included studies $(n=60$ of 108$)$ reported at least one component in all three categories (see online supplementary appendix 2). This is concerning as diagnostic imaging at this point in time lacks consensus on the specific modalities and cut-off values necessary for this intervention.

The utilisation of at least one diagnostic imaging modality was reported in $92 \%$ of studies in this review. Findings suggesting a significant emphasis on diagnostic imaging are in line with previous research. ${ }^{9}$ While diagnostic imaging, especially utilisation of alpha angle as found in this review, is one of the most objective and standardised procedures to guide the treating surgeon, reliance on imaging alone should be cautioned since the correlation between cam morphology and hip pain is imprecise. ${ }^{614}$ Additionally, 37\% of asymptomatic individuals have cam morphology while $67 \%$ have pincer morphology recent findings. ${ }^{15}$ Precise diagnostic values to define cam and pincer morphology have not been recommended, due to the complexity of the relationship between morphology and symptoms. Diagnostic imaging has been recommended as a necessary component of comprehensive approach to the diagnosis of FAIS though. ${ }^{3}$ Perhaps of greater concern for the diagnosis of FAIS are the eight studies included in this review that did not utilise diagnostic imaging at all. Therefore, while diagnostic imaging can be a valuable tool for the surgeon in diagnosing and treating FAIS, currently, there is still significant variability in utilisation and interpretation of such findings.

Only 30\% reported limited range of motion as a surgical criterion. The relevance of limited range of motion in those with FAIS is still unclear. ${ }^{16}{ }^{17}$ A correlation between limited hip internal rotation range of motion and cam-type morphology has been suggested, although all of these subjects were asymptomatic. ${ }^{141819}$ High prevalence of limited hip internal rotation in American football players ${ }^{18}$ and external rotation range of motion in hockey players ${ }^{20}$ might even suggest a normal sport adaptation rather than pathology. Thus, limitations in range of motion should be reported consistently in surgical studies to ascertain its relationship to FAIS.

Failed non-surgical care as a criterion for surgery was reported in less than half of the studies in our review. Specific detail regarding the non-surgical intervention that was employed (eg, 
NSAIDs, activity modification and physical therapy) was only reported in $25 \%$ of these studies. Only $18 \%$ of studies specifically mentioned physiotherapy as a trialled intervention. A trial of non-surgical care has been suggested (unless contraindicated) for clients with FAIS prior to surgical intervention, ${ }^{1021}$ although it is worthy of mention that non-surgical treatment evidence for FAI is limited to level IV studies and is poorly reported. ${ }^{921}$ Future randomised controlled trials are essential to determine the efficacy of physiotherapy for treatment of FAIS.

Duration of symptoms was reported in only $30(28 \%)$ studies, with exactly half of these studies reporting 3 months or less. While it is important to seek early intervention in the care of musculoskeletal conditions, especially to avoid excessive healthcare utilisation prior to FAIS diagnosis, ${ }^{722}$ it is also known that many painful conditions will resolve or improve without intervention. ${ }^{23}{ }^{24}$ Therefore, while it is quite possible the participants in these studies did potentially meet this guideline of hip pain $>3$ months, this should be reported in the studies.

This scoping review highlights the wide variety of studies that were included. Incorporating all studies where patients underwent surgery for FAIS, rather than including only outcomes studies, more accurately reflects the current status of FAIS diagnosis and treatment findings, enlightening clinicians and researchers to the current variability in inclusion criteria for the surgical treatment of FAIS. This type of review is ideal in disciplines with emerging evidence, as is the case with FAIS research, because it allows researchers to address questions beyond those related to intervention effectiveness. ${ }^{25}$

This review reported surgical criteria according to recent standards for the diagnosis of FAIS. ${ }^{313}$ It is worthy of mention that these standards, especially the 2016 Warwick Agreement on FAIS, ${ }^{3}$ are new and would not have been available when designing previous studies. However, these standards should reflect current standards for study design and reporting.

\section{Limitations}

This review is not without limitations. One major limitation of this study was that conclusions were made based only on what was reported in the included studies. Some objective measures, such as range of motion, were poorly defined, with $11 \%$ of studies reporting range of motion as 'limited', 'decreased' or 'measured'. Another limitation was that only studies published in the English language were included. Furthermore, due to the fact that this was a scoping review and not a systematic review, a quality assessment of the studies was not performed.

\section{CONCLUSIONS}

As hypothesised, diagnostic imaging evidence of FAIS was the most commonly reported criterion for FAIS surgery $(92 \%$ of included studies). Only 56\% of included studies utilised the combination of symptoms, clinical signs and diagnostic imaging for diagnosis of FAI syndrome as suggested by the Warwick Agreement on FAIS meeting. The most commonly reported surgical criteria in these $56 \%$ of articles reporting all three areas were symptomatic hip pain of greater than 6 months' duration (symptom), decreased hip flexion and internal rotation range of motion (clinical sign), a positive impingement sign (clinical sign), alpha angle greater than $50^{\circ}$ (diagnostic imaging) and a positive crossover sign (diagnostic imaging). Interestingly, failed non-surgical treatment (only $44 \%$ of all studies) and failed physiotherapist-led rehabilitation (only 18\% of all studies) were not commonly utilised in the surgical decision-making process for patients with FAIS. Only $12 \%$ utilised intra-articular injection as
What are the findings?

- The most commonly reported criteria for FAIS surgery is diagnostic imaging, despite a lack of consensus on the specific modalities and cut-off values necessary for this intervention.

- In only $56 \%$ of FAIS surgery studies, the criteria for surgery included a combination of symptoms, clinical signs and diagnostic imaging as suggested by recent consensus findings.

- Failed non-surgical treatment (44\% of included studies), failed physiotherapist-led rehabilitation ( $18 \%$ of included studies) and relief of pain from intra-articular injection (12\% of included studies) could be relevant criteria to determine readiness for surgical intervention in patients diagnosed with FAIS.

\section{How might it impact on clinical practice in the future?}

- The tendency towards primary reliance on diagnostic imaging and limited reliance on a comprehensive diagnosis of FAIS syndrome (patient report of symptoms, clinical signs and diagnostic imaging) for surgical correction, may result in inappropriate patient selection.

- Future FAIS surgical studies would benefit from criteria for surgery that includes a combination of symptoms, clinical signs and diagnostic imaging.

- These studies should also consider including failed non-surgical treatment or physiotherapist-led rehabilitation as well as intra-articular diagnostic injection as part of the decision-making concerning performing FAIS surgery or not.

a surgical criterion for FAIS diagnosis. Specific surgical criteria, as well as their specific parameters and values, demonstrated major inconsistencies across the studies, making it difficult to ascertain patient characteristics of the FAIS patient.

Acknowledgements We would like to thank Leila Ledbetter, MLIS, for assisting withthe literature search for this study

Contributors MPR contributed idea of manuscript, initial registration of review, initial search, review of data, initial drafting and editing of manuscript. SP contributed initial search, data extraction and editing of manuscript. AL, CE, KM and TJ contributed initial search and data extraction. KT and PH contributed review and editing of manuscript. All authors approved the final draft of the manuscript.

Competing interests None declared.

Provenance and peer review Commissioned; externally peer reviewed.

Data sharing statement Data available on request.

(c) Article author(s) (or their employer(s) unless otherwise stated in the text of the article) 2017. All rights reserved. No commercial use is permitted unless otherwise expressly granted.

\section{REFERENCES}

1 Ganz R, Parvizi J, Beck M, et al. Femoroacetabular impingement: a cause for osteoarthritis of the hip. Clin Orthop Relat Res 2003;417:112-20.

2 Reiman MP, Thorborg K. Femoroacetabular impingement surgery: are we moving too fast and too far beyond the evidence? Br J Sports Med 2015;49:782-4.

3 Griffin DR, Dickenson EJ, O'Donnell J, et al. The warwick agreement on femoroacetabular impingement syndrome (FAl syndrome): an international consensus statement. Br J Sports Med 2016;50:1169-76.

4 Lavigne M, Parvizi J, Beck M, et al. Anterior femoroacetabular impingement: part I. techniques of joint preserving surgery. Clin Orthop Relat Res 2004 418:61-6. 
5 Beck M, Kalhor M, Leunig M, et al. Hip morphology influences the pattern of damage to the acetabular cartilage: femoroacetabular impingement as a cause of early osteoarthritis of the hip. J Bone Joint Surg Br 2005;87:1012-8.

6 Dickenson E, Wall PD, Robinson B, et al. Prevalence of cam hip shape morphology: a systematic review. Osteoarthritis Cartilage 2016;24:949-61.

7 Cvetanovich GL, Chalmers PN, Levy DM, et al. Hip arthroscopy surgical volume trends and 30-day postoperative complications. Arthroscopy 2016;32:1286-92.

8 Khan M, Ayeni OR, Madden K, et al. Femoroacetabular impingement: Have we hit a global tipping point in diagnosis and treatment? results from the InterNational femoroacetabular impingement optimal care update survey (IN FOCUS). Arthroscopy 2016;32:779-87.

9 Ayeni OR, Wong I, Chien T, et al. Surgical indications for arthroscopic management of femoroacetabular impingement. Arthroscopy 2012;28:1170-9.

10 Reiman MP, Thorborg K, Hölmich P. Femoroacetabular impingement surgery is on the risee-but what is the next step? J Orthop Sports Phys Ther 2016;46:406-8.

11 Arksey H, O'Malley L. Scoping studies: towards a methodological framework. Int I Soc Res Methodol 2005;8:19-32.

12 Murray A, Daines L, Archibald D, et al. The relationship and effects of golf on physical and mental health: a scoping review protocol. Br I Sports Med 2016:50:647-50.

13 Nepple JJ, Prather H, Trousdale RT, et al. Clinical diagnosis of femoroacetabular impingement. J Am Acad Orthop Surg 2013;21(Suppl 1):S16-S19.

14 Tak I, Glasgow P, Langhout R, et al. Hip range of motion is lower in professional soccer players with hip and groin symptoms or previous injuries, Independent of cam deformities. Am I Sports Med 2016;44:682-8.

15 Frank JM, Harris JD, Erickson BJ, et al. Prevalence of femoroacetabular impingement imaging findings in asymptomatic volunteers: a systematic review. Arthroscopy 2015;31:1199-204.
16 Audenaert EA, Peeters I, Vigneron L, et al. Hip morphological characteristics and range of internal rotation in femoroacetabular impingement. Am I Sports Med 2012;40:1329-36.

17 Freke MD, Kemp J, Svege I, et al. Physical impairments in symptomatic femoroacetabular impingement: a systematic review of the evidence. Br J Sports Med 2016;50:1180.

18 Kapron AL, Anderson AE, Peters CL, et al. Hip internal rotation is correlated to radiographic findings of cam femoroacetabular impingement in collegiate football players. Arthroscopy 2012;28:1661-70.

19 Yuan BJ, Bartelt RB, Levy BA, et al. Decreased range of motion is associated with structural hip deformity in asymptomatic adolescent athletes. Am I Sports Med 2013:41:1519-25.

20 Lerebours F, Robertson W, Neri B, et al. Prevalence of cam-type morphology in elite ice hockey players. Am J Sports Med 2016;44:1024-30.

21 Wall PD, Fernandez M, Griffin DR, et al. Nonoperative treatment for femoroacetabular impingement: a systematic review of the literature. PM R 2013;5:418-26.

22 Keeney JA, Peelle MW, Jackson J, et al. Magnetic resonance arthrography versus arthroscopy in the evaluation of articular hip pathology. Clin Orthop Relat Res 2004:429:163-9.

23 Cassidy JD, Côté P, Carroll LJ, et al. Incidence and course of low back pain episodes in the general population. Spine 2005;30:2817-23.

24 Manchikanti L, Singh V, Falco FJ, et al. Epidemiology of low back pain in adults. Neuromodulation 2014;17(Suppl 2):3-10.

25 Levac D, Colquhoun H, O'Brien KK. Scoping studies: advancing the methodology. Implement Sci 2010;5:69. 